\title{
Twin paradox in curved spacetime
}

\author{
E. Benedetto ${ }^{* 1,2 @}$, A. Feoli ${ }^{2 @}$, A. L. Iannella ${ }^{2}$ \\ ${ }^{1}$ Università Degli Studi di Salerno, Via Giovanni Paolo II, 132, 84084 Fisciano SA, Italia. \\ ${ }^{2}$ Università Degli Studi del Sannio, Dipartimento di Ingegneria, Piazza Roma, 21, 8210 Benevento, Italia.
}

Received on September 16, 2021. Accepted on January 25, 2022.

\begin{abstract}
The twin paradox has played an important role in the history of special relativity (SR). A precise calculation would require the application of the general theory of relativity (GR) but, neglecting the acceleration phases of the traveling twin, even in SR it is possible to find the correct solution without logical contradictions. Nowadays it is well known that the twin thought experiment seems a paradox as a consequence of a naive application of time dilation and the principle of relativity. The twin who goes on space travel is the one who, returning to the twin at rest, finds the aged brother. Continuing in this pedagogical tradition, we want to add a further consideration. In fact, the experiment has always been explained by considering the two twins immersed in Minkowski's spacetime. It could be interesting, from a didactic point of view, to analyze the same thought experiment without neglecting the Earth's gravitational field. Indeed, under certain conditions, it may happen that the traveling brother can grow older than his brother at rest.
\end{abstract}

Keywords: Twin paradox; gravitational time dilation; general relativity.

\section{Introduction}

Very often, many things that are obvious for specialists are not so obvious for students and it is useful, from a didactic point of view, to investigate some aspects of a topic [1] 4. For example, the twin paradox is a very famous thought experiment in SR [5-7]. Twin $A$ stays on Earth and $B$ takes a journey at relativistic speed and then returns finding that $A$ has aged more. This result seems paradoxical, because each twin, by the principle of relativity, can be considered at rest, and therefore, each of them should find the other who has aged less. Considering instantaneous accelerations and decelerations, it is possible to have a precise solution in SR [8], even if, for an exact treatment, it is necessary to apply GR [9]. The discussion of how you get the correct interpretation in SR is not the aim of this paper. We would just like to mention that the link between the times measured by the two twins is

$$
\frac{\Delta t_{A}}{\Delta t_{B}}=\gamma=\frac{1}{\sqrt{1-\frac{v^{2}}{c^{2}}}} \approx 1+\frac{v^{2}}{2 c^{2}}>1
$$

For this reason, we have always $\Delta t_{A}>\Delta t_{B}$ and it means that the time is longer for the twin on Earth. What happens if we do not neglect the Earth's gravitational field? In this case, the gravitational potential affects proper time intervals and also the spatial intervals. So, in these situations, we have the algebraic sum between a kinematic and a gravitational effect.

\footnotetext{
${ }^{*}$ Correspondence email address: elmobenedetto@libero.it
}

\section{Twin paradox in curved spacetime}

For simplicity of calculation, since this is a paper with an educational purpose, let us consider $v \ll c$ as, for example, in the well known Hafele-Keating experiment and also the following weak field metric [9]

$$
\begin{aligned}
d s^{2}= & \left(1-\frac{2 G M}{c^{2} r}\right) c^{2} d t^{2}-\left(1+\frac{2 G M}{c^{2} r}\right) d r^{2} \\
& -r^{2}\left(d \theta^{2}+\sin ^{2} \theta d \phi^{2}\right)
\end{aligned}
$$

where $\theta$ and $\phi$ are the usual polar and azimuthal angles, $t$ is the time coordinate measured by a stationary clock located infinitely far from the massive body, $r=$ $\frac{C}{2 \pi}$ where $C$ is the circumference of a circle centered on the Earth. Finally, obviously, $G$ is the Newtonian gravitational constant, $M$ is the mass of the Earth and $c$ is the speed of light. With these starting hypotheses, we can safely neglect the infinitesimals of higher order than $V / c^{2}$ where $V$ is the gravitational potential. Twin $A$ stays on Earth and $B$, remaining in a region where the presence of the gravitational field is always not negligible, takes a journey in radial direction at speed $v$ along a path of length $l$ arriving at the position $r_{2}$, and then returns. For an external observer, far from the gravitational field, we have

$$
\Delta t=\frac{2 l}{v} .
$$

Obviously, the velocity is calculated with respect to the coordinate system $(r, \theta, \phi, t)$. Furthermore, we are neglecting the motions of rotation and revolution of the 
Earth. For the twin who remains on Earth we have the proper time [10]

$$
\Delta \tau_{A}=\sqrt{g_{00}\left(r_{E}\right)} \Delta t \approx\left(1-\frac{G M}{c^{2} r_{E}}\right) \frac{2 l}{v},
$$

where $r_{E}$ is the Earth's radius. We have that

$$
l \approx \int_{r_{E}}^{r_{2}}\left(1+\frac{G M}{c^{2} r}\right) d r=r_{2}-r_{E}+\frac{G M}{c^{2}} \ln \frac{r_{2}}{r_{E}}
$$

getting

$$
\Delta \tau_{A} \approx \frac{2}{v}\left(1-\frac{G M}{c^{2} r_{E}}\right)\left(r_{2}-r_{E}+\frac{G M}{c^{2}} \ln \frac{r_{2}}{r_{E}}\right) .
$$

We can also write the previous relation in the following way, which will be useful later

$\Delta \tau_{A} \approx \frac{2}{v}\left(1-\frac{G M}{c^{2} r_{E}}\right)\left(r_{2}-r_{E}\right)\left(1+\frac{G M}{\left(r_{2}-r_{E}\right) c^{2}} \ln \frac{r_{2}}{r_{E}}\right)$.

For the twin $B$, we have the proper time

$$
d \tau_{B}^{2}=\frac{d s^{2}}{c^{2}}=\left(1-\frac{2 G M}{c^{2} r}\right) d t^{2}-\left(1+\frac{2 G M}{c^{2} r}\right) \frac{d r^{2}}{c^{2}} .
$$

By neglecting the infinitesimals of higher order, we can write

$$
d \tau_{B}^{2}=\left(1-\frac{2 G M}{c^{2} r}-\frac{v^{2}}{c^{2}}\right) d t^{2}
$$

finally getting

$$
d \tau_{B}=\sqrt{1-\frac{2 G M}{c^{2} r}-\frac{v^{2}}{c^{2}}} d t \approx\left(1-\frac{G M}{c^{2} r}-\frac{v^{2}}{2 c^{2}}\right) d t .
$$

Therefore

$$
\Delta \tau_{B}=\frac{2}{v} \int_{r_{E}}^{r_{2}}\left(1-\frac{G M}{c^{2} r}-\frac{v^{2}}{2 c^{2}}\right)\left(1+\frac{G M}{c^{2} r}\right) d r .
$$

By neglecting, even now, the infinitesimals of higher order

$$
\Delta \tau_{B} \approx \frac{2}{v} \int_{r_{E}}^{r_{2}}\left(1-\frac{v^{2}}{2 c^{2}}\right) d r=\frac{2}{v}\left(r_{2}-r_{E}\right)\left(1-\frac{v^{2}}{2 c^{2}}\right)
$$

From (7) and $\sqrt{12}$ we obtain

$$
\frac{\Delta \tau_{A}}{\Delta \tau_{B}}=\frac{\left(1-\frac{G M}{c^{2} r_{E}}\right)\left(1+\frac{G M}{\left(r_{2}-r_{E}\right) c^{2}} \ln \frac{r_{2}}{r_{E}}\right)}{1-\frac{v^{2}}{2 c^{2}}} .
$$

Neglecting the infinitesimals of higher order with respect to $G M / c^{2} r$, it is possible to write

$$
\frac{\Delta \tau_{A}}{\Delta \tau_{B}} \approx\left(1+\frac{v^{2}}{2 c^{2}}\right)\left(1+\frac{G M}{\left(r_{2}-r_{E}\right) c^{2}} \ln \frac{r_{2}}{r_{E}}-\frac{G M}{c^{2} r_{E}}\right),
$$

finally getting

$$
\frac{\Delta \tau_{A}}{\Delta \tau_{B}} \approx 1+\frac{v^{2}}{2 c^{2}}-\frac{G M}{c^{2} r_{E}}+\frac{G M}{\left(r_{2}-r_{E}\right) c^{2}} \ln \frac{r_{2}}{r_{E}} .
$$

Therefore, we have the kinematic term $1+\frac{v^{2}}{2 c^{2}}$, as it happens in flat spacetime, but also a gravitational contribution. So, in Minkowski's spacetime, it is always $\Delta \tau_{A}>\Delta \tau_{B}$ while, in curved spacetime, $\frac{\Delta \tau_{A}}{\Delta \tau_{B}}>1$ if and only if

$$
v^{2}>\frac{2 G M}{r_{E}}-\frac{2 G M}{\left(r_{2}-r_{E}\right)} \ln \frac{r_{2}}{r_{E}}
$$

\section{Conclusion}

In this paper, we have considered the twin paradox in the presence of gravity. This thought experiment, being generally treated in SR, assumes that the twins are in two inertial frames of Minkowski spacetime. Having a didactic purpose, we have simplified the formalism, by considering a weak gravitational field and nonrelativistic velocities. Even in this simplified case, we were able to show how in curved spacetime the youngest twin is not always the travelling one.

\section{Acknowledgments}

This research was partially supported by FAR fund of the University of Salerno.

\section{References}

[1] D.V. Redzic, European Journal of Physics 29, 191 (2008).

[2] D.V. Redzic, European Journal of Physics 39, 025205 (2018).

[3] E. Benedetto and A. Feoli, European Journal of Physics 38, 055601 (2017).

[4] A. Feoli and E. Benedetto, Physics Education 56, 015002 (2021).

[5] R. Resnick, Introduction to special relativity (John Wiley \& Sons Inc, New Jersey, 1968).

[6] J.A. Wheeler, A journey into gravity and spacetime (W.H. Freeman \& Company, New York, 1990).

[7] P. Davies, About Time: Einstein's Unfinished Revolution (Penguin Books, Simon \& Schuster, New York, 1995).

[8] W. Pauli, Theory of Relativity (Dover Publications, New York, 1958).

[9] H.C. Ohanian and R. Ruffini, Gravitation and Spacetime (Cambridge University Press, Cambridge, 2013).

[10] L.D. Landau and E.M. Lifshitz, The Classical Theory of Fields, Course of Theoretical Physics (Pergamon Press, Oxford, 1984). 